advancement of the disease-and in particular to spread outside the thyroid gland and infiltration of the trachea. The behaviour of these tumours is influenced by the presence and not the proportion of the papillary component. Permanent hormone replacement therapy is mandatory: it will suppress pituitary production of thyroid stimulating hormone (TSH) and may cause regression of metastases.

Follicular tumours account for $15 \%$ of all cases. They are often found in longstanding goitres and are rare before the age of 30 . Spread to the lymph nodes is not common, but extrathyroidal infiltration is frequent. There is often dissemination by the blood stream, causing pulmonary or osseous metastases, but these may not be present until many years after the treatment of the primary tumour. The survival time is disappointing and reflects the failure of inadequate surgery. ${ }^{9}$

At operation the affected lobe should be removed and if the diagnosis can be confirmed total thyroidectomy performed together with removal of any visible lymph nodes. ${ }^{4}$ If no firm diagnosis can be made at operation the remaining lobe may need to be removed later or ablated with radioiodine. These tumours respond well to a combination of surgery and radioisotope treatment, and thyroidectomy should be advised even if there is evidence of disseminated disease.

In all cases of papillary and follicular tumours, once surgical treatment is complete, a programme of neck and whole body scanning should routinely follow before replacement thyroxine treatment is started. An ablative dose of ${ }^{131}$ I should be given to eliminate any residual normal thyroid tissue in the neck. Replacement therapy with T3 (triiodothyronine) should then be given for six weeks. Ten days after treatment with T3 has stopped both the neck and the whole body should be scanned: if there is uptake of tracer in the neck or elsewhere, a therapeutic dose of ${ }^{131}$ I should be given; treatment with T3 should then be resumed for another three months and scanning repeated. If a tracer dose shows uptake further therapeutic doses will need to be given until the metastases are totally eradicated or treatment fails. Only when all therapeutic or diagnostic procedures are complete should the patient be given replacement therapy with $\mathrm{T} 4$ (thyroxine).

Medullary tumours, with their amyloid stroma, arise from the parafollicular or C-cells and form less than $10^{\circ}$ of the total of all thyroid cancers. They are mainly seen in patients aged over 30 and are often bilateral; they may be familial, while metastasis to the lymph nodes and further dissemination are common. Calcitonin assays may be used to confirm the diagnosis both in the patient and in family members at risk; repeat assays during follow-up will indicate the presence of extrathyroidal spread or the development of metastases. Total thyroidectomy with removal of affected nodes is the operation of choice, provided the parathyroids can be conserved. With adequate surgery ${ }^{10}$ the recurrence rate is $23 \%$ and the ten-year survival $27 \% .{ }^{131} \mathrm{I}$ has no place in treatment, but supervoltage radiotherapy does appear to delay the progress of local recurrence.

In many cases anaplastic tumours (which form $15 \%$ of the total) may arise as a mutation from a differentiated tumour. This may account for their occurrence in elderly patients with a longstanding goitre which suddenly grows rapidly, becomes painful, and causes pressure symptoms. The clinical diagnosis usually presents no problems. The surgical plan should be removal of as much tumour as possible. This type does not concentrate ${ }^{131} \mathrm{I}$ and so radioiodine has no place in the postoperative management. High-dosage supervoltage external beam radiotherapy should therefore be given to delay the onset of recurrence and obstructive symptoms. Combination chemotherapy may be tried, but it is only rarely successful.
Childhood thyroid cancer is a separate entity. The average age at presentation is between 7 and 10 years. There is usually a long history of painless persistent cervical lymphadenopathy or a goitre, and the possibility of a neoplasm has rarely been considered. Histologically, most tumours are papillary and the rest follicular or anaplastic. The survival prospects are good when the tumours are differentiated and treatment is started early. Because of the risk of permanent hypoparathyroidism total thyroidectomy is not indicated. ${ }^{11}$

${ }^{1}$ Williams, E D, British fournal of Surgery, 1975, 62, 757.

2 Staunton, M D, and Greening, W P, British Medical fournal, 1973, 4, 532

${ }^{3}$ Hirabayashi, R N, and Stuart, Lindsay, fournal of Clinical Endocrinology, $1961 / 21,1596$

4 Black, B M, YaDean, R E, and Wollner, L B, Archives of Surgery, 1964, 88, 610

${ }^{5}$ Greening, W P, in Tumours of the Thyroid Gland, ed D Smithers, p 231. Edinburgh and London, Churchill Livingstone, 1974.

${ }^{6}$ Crile, G, Jr, Surgery, Gynecology, and Obstetrics with International Abstracts of Surgery, 1971, 132, 460.

'Clark, R L, Ibanez, M L, and White, E C, Archives of Surgery, 1966, 92, 23.

${ }^{8}$ Marchetta, F C, fournal of Surgical Oncology, 1974, 6, 401.

9 Staunton, M D, and Martin, M, in Tumours of the Thyroid Gland, ed D Smithers, p 276. Edinburgh and London, Churchill Livingstone, 1974.

${ }^{10}$ Chong, Guan C, et al, Cancer, 1975, 35, 695.

${ }^{11}$ Buckwalter, J A, Thomas, C G, and Freeman, J B, Annals of Surgery, $1975,181,632$.

\section{Maternal mortality}

With the publication of the $1970-2$ report, ${ }^{1}$ Confidential Enquiries into Maternal Deaths in England and Wales now cover 21 years. This one is as valuable as its predecessors, all of which have contributed something to the practice of obstetrics and lowering maternal mortality-evident over the years and still continuing. Careful statistical analysis based on good data has highlighted women "at risk" so that their care has been intensified. Some of those who might previously have died have been saved. Standards of practice have so improved, partly as a result of the reports, that in some chapters the defined "avoidable factors" have shown a percentage increase, though the numbers of patients dying from particular conditions have greatly decreased.

A maternal death is one occurring during pregnancy, during labour, or as a consequence of pregnancy and within one year of delivery or abortion. (The international definition takes into account only deaths within 42 days of delivery or abortion.) The report is concerned with 606 maternal deaths in England and Wales in the three-year period 1970-2; of these, 355 were directly due to pregnancy or childbirth and 251 were from associated causes. In fact, the Registrar-General recorded more than 606 deaths in these three years, but this figure represents $91^{\circ}$ of his total, so it appears that the method pursued in gathering the information for the confidential reports has become acceptable to those concerned in the misfortune of a maternal death.

The decline in the numbers of deaths is not simply due to the falling birth rate occasioned by contraception and abortion, for the mortality rate per 1000 births (including deaths due to abortion) fell from 0.67 in 1952 to 0.16 in 1972 . Social changes have probably been partly responsible for these better results; these include a reduction in the number of births to women aged over 30 and to those having five or more children. Also of some significance is the decreased number of women being confined at home and in nursing homes. In $197290 \%$ of all 
births were in NHS hospitals. Social patterns and the provision of obstetric services may explain why the maternal mortality rate (excluding abortions) ranges from 5.9 in the Oxford region to $17 \cdot 2$ in the Leeds region with many gradations between these two. The Metropolitan figures vary from 12.7 to 16.0 per 100000 total births. Oxford shows what is attainable. The reasons for these regional differences should be analysed more carefully, and the findings made the basis of action.

Most deaths were due to the old ogres-abortion (81), pulmonary embolism (61), toxaemia (47), ectopic pregnancy (34), sepsis excluding abortion (32), haemorrhage (27), amniotic fluid embolism (22), and ruptured uterus (12), and there was a miscellaneous group of 178. Though there has been some improvement in nearly all areas, there are the same familiar human failures. It was thought that there were avoidable factors in 347 of the deaths: roughly $31^{\circ}{ }^{\circ}$ attributed to patients, $42^{\circ}$ o to hospital staff, $17^{\circ}{ }_{\circ}$ to general practitioners, $8^{\circ}{ }_{0}$ to anaesthetists, $2^{\circ}$ to midwives, and $0.3^{\circ}{ }_{0}$ to administration. Something can still be done to increase medical awareness of danger, and there is still a need for education of patients. Recurring indictments are of consultants unwisely delegating obstetric and anaesthetic care to those not yet competent to discharge the responsibility and of general practitioners not recognising warning signs. Control of haemorrhage has vastly improved but there are still failures to recognise the potential dangers of placenta praevia. Pulmonary embolism has been declining as a cause of death, but the reason is not elucidated. Phaeochromocytoma claimed four lives. Anaesthetic deaths were fewer than previously, but of the 35 cases there was probably an avoidable factor in 31 . The dangers of ectopic pregnancy do not seem to be fully grasped-there was an avoidable factor in 11 of 34 deaths.

All who have a care for pregnant women have a duty to read this new report-and to mark and inwardly digest it. The return for the effort may well be that some childbearing woman's life may be saved in the future because dangers were understood and acted upon.

${ }^{1}$ Report on Confidential Enquiries in Maternal Deaths in England and Wales 1970-2. Lodon, HMSO, 1975.

\section{Obituary for fascia lata heart valves}

When Senning ${ }^{1}$ first described valves fabricated from fascia lata for replacing the aortic valve it seemed as if a great advance had been made. Here was the ideal valve substitute: it had a central flow orifice, was non-obstructive, free from the problems of thromboembolism, and was made from strong durable autologous living tissue which was readily available in assorted sizes. Senning's next report, ${ }^{2}$ dealing with his first 90 operations, was encouraging - though eight patients had developed moderate or severe aortic regurgitation and most had an early diastolic murmur. There was also a disquieting incidence of late endocarditis.

The technique was adopted enthusiastically in centres on both sides of the Atlantic, but little attention was paid to the reports $^{3-5}$ of experimental work on fascial valve replacement which began to appear about this time. These emphasised a high incidence of valve failure. Pathological studies showed the development of fibrosis, thickening, degeneration, and cal- cification of the material, with fusion of commissures and retraction of cusps leading to valve incompetence even as early as two months after insertion.

In 1969 a method of fixation of the fascia lata valve to a rigid frame was described, ${ }^{6}{ }^{7}$ so that it became possible to replace any cardiac valve with a fascial valve. Optimistic short-term accounts of both aortic and mitral valve replacement appeared, ${ }^{6}{ }^{8}$ but by 1971 doubts had arisen about the durability of the material-at any rate in the mitral position. In 1972 McEnany, Ross, and Yates $^{9}$ reported short-term results in 72 frame-supported autologous fascia lata valves. Valve failure occurred in $60^{\circ}$ of the survivors of the operation, and $23 \%$ of the original patients had died within two years, most of them from mitral regurgitation. Having found reasonably good function in fascial aortic valves and failure in the tricuspid and mitral positions, this group suggested that it was haemodynamic factors giving rise to irregular stress on the cusps in the atrioventricular site that led to thickening and shrinkage.

By 1973 Senning had inserted 200 fascial aortic valves. A follow-up of 84 survivors for between five and ten years ${ }^{10}$ showed that one in six had developed endocarditis on the fascial valve, most commonly between two and eight years after operation. Aortic incompetence progressively increased from $15 \%$ at three and a half years to $42 \%$ at five years. Only three patients had no regurgitation, and only one-third had good function. One-quarter of the patients had died, from either endocarditis or valvular insufficiency. Fibrosis, calcification, and the formation of cartilage were seen in the fascia.

Recent reports on the long-term results of fascial valve replacement both in the mitral ${ }^{11}{ }^{12}$ and aortic ${ }^{13}$ positions have confirmed these findings. The fascial mitral valve shows progressive deterioration with regurgitation over $12-18$ months, and at three years only $5-10 \%$ remain competent. Late endocarditis and progressive regurgitation after five years characterise the aortic fascial valves, one-third of which require reoperation. After eight years some valves become stenotic and calcified. ${ }^{14}$

In 1970 an international meeting on fascia lata heart valves was held in Toronto, ${ }^{15}$ which decided to set up a cooperative international study and a central registry. The pathologists in this project have now examined 58 excised fascia lata valves which had been in situ for up to 50 months. ${ }^{16}$ The early pseudointima had become fibrotic, leading to shrinkage; the cells of the original fascia had disappeared, and the material had ultimately died, with vacuolation and late calcification; cusps had become thickened from organisation of the surface thrombus, and the subsequent shrinkage had led to incompetence of the valves irrespective of the site of implantation. Olsen ${ }^{17}$ found similar changes in 16 framemounted valves. His viability studies showed greatly reduced activity: there was degeneration of the collagen, a decrease in the number of fibroblasts, and superimposition of fibrin on the fibrous tissue. The shrinkage was due to contraction of this fibrous tissue, which sandwiched the fascial cusps, and the changes progressed with time.

Despite the adverse evidence the idea of the dead fascial valve periodically comes back into favour. Trimble, ${ }^{18}$ reviewing the results of 36 fascial aortic valve replacements at three and a half years, decided that they were sufficiently satisfactory to justify returning to their use; and Ionescu, ${ }^{19}$ while agreeing that autologous fascia lata is unsuitable, has recently made a case for the use of preserved heterologous fascia (on the basis of a small series) and for heterologous pericardium (for which his follow-up is between one and three years.)

When writing the obituary of the fascia lata valve one must have an epitaph for its tombstone. It is to be found in a paper 